\title{
Money Circulation Equation Considering Time Irreversibility
}

\author{
Shinji Miura \\ Independent, Gifu, Japan \\ Email: geppa_gifu@yahoo.co.jp
}

Received 29 August 2014; revised 30 September 2014; accepted 31 October 2014

Copyright @ 2014 by author and Scientific Research Publishing Inc.

This work is licensed under the Creative Commons Attribution International License (CC BY). http://creativecommons.org/licenses/by/4.0/

(c) (i) Open Access

\section{Abstract}

The essence of money circulation is that money continues to transfer among economic agents eternally. Based on this recognition, this paper shows a money circulation equation that calculates the quantities of expenditure, revenue, and the end money from the quantity of the beginning money. The beginning money consists of the possession at term beginning, production and being transferred from the outside of the relevant society. The end money consists of the possession at term end, disappearance and transferring to the outside of the relevant society. This equation has a unique solution if and only if each part of the relevant society satisfies the space-time openness condition. Moreover, if money is transferred time irreversibly, each part of the relevant society satisfies the space-time openness condition. Hence, the solvability of the equation is guaranteed by time irreversibility. These solvability conditions are similar to those of the economic inputoutput equation, but the details are different. An equation resembling our money circulation equation was already shown by Mária Augustinovics, a Hungarian economist. This paper examines the commonalities and differences between our equation and hers. This paper provides the basis for some intended papers by the author.

\section{Keywords}

Money Circulation, Solvability, Time Irreversibility, Mária Augustinovics

\section{Introduction}

Simon Newcomb, who was an American economist in the nineteenth century, said:

"A piece of money changes hands without end, since every person who receives it expects, unless in exceptional cases, to pay it out again to some one else."

${ }^{1}$ Newcomb [1] p. 315. 
As Newcomb said, money received once cannot be transferred again in principle. As a result, money continues to transfer among economic agents eternally. This is the essence of money circulation.

In order to express the quantitative structure of money circulation, the above mentioned Newcomb used the equation of exchange, which he called the equation of societary circulation. ${ }^{2}$ However, the relationship between the velocity of money, which is the key concept of this equation, and the situations of each agent is not clear. In other words, the velocity of money is an enigma. To formalize a money circulation equation which remedies this defect is a purpose of this paper.

Although describing optimal decision making for an economic agent is a mainstream in contemporary economics, this paper does not adopt such a method. This paper describes only a realized situation of money circulation. This paper aims to construct a kinematics of money.

Then, we will start the description of a monetary economy.

We decide that a target group for description is called the relevant society. The element of the relevant society is called an economic agent. ${ }^{3}$ There are various kinds of agents, such as an individual, a family, a corporation, the government, and so on. However, in this paper, we do not consider the differences in such kinds of agents. We treat them merely as agents in the group. We assume that there are $n$ agents in the relevant society, and denote the relevant society by $N=\{1,2, \cdots, n\}$.

Moreover, we decide that a target term for description is called the relevant term. We assume that the relevant term is finite. This assumption is rewritten so that the relevant term always has a beginning and an end. This assumption does not threaten the validity of the description of an economic society because most scientists say even the life of the earth is limited.

The sphere that satisfies both the relevant society and the relevant term is called the relevant space-time.

There are various kinds of money transfer, including purchase of commodity, payment of wage, finance relation, donation, and so on. However, in this paper, we do not consider such differences. We treat them as merely transfers in the group.

Moreover, there are various kinds of money such as the dollar, pound, euro, yen, and so on. However, in this paper, we assume that money in the relevant space-time is of only one kind. ${ }^{4}$

We define expenditure as transferring money to the relevant space-time, and revenue as money being transferred from the relevant space-time. There is a possibility that a transfer of money occurs between the relevant society and the outside of there, but transferring money to the outside is not called expenditure and money being transferred from the outside is not called revenue in this paper.

Let $X_{i}$ be the expenditure quantity of Agent $i, Y_{j}$ be the revenue quantity of Agent $j$, and $Z_{i j}$ be the separate transfer quantity from Agent $i$ to Agent $j$. By the definition of expenditure and revenue which is mentioned above, the following relations are satisfied.

$$
\begin{array}{cc}
Z_{i 1}+Z_{i 2}+\cdots+Z_{i n}=X_{i} & \text { for } \forall i \in N \\
Z_{1 j}+Z_{2 j}+\cdots+Z_{n j}=Y_{j} & \text { for } \forall j \in N
\end{array}
$$

The relationships between expenditures, revenues and separate transfers can be summarized as in the following table.

\begin{tabular}{cccccc}
\hline & Agent 1 Revenue & Agent 2 Revenue & $\ldots$ & Agent $n$ Revenue & Sum \\
\hline Agent 1 Expenditure & $Z_{11}$ & $Z_{12}$ & $\ldots$ & $Z_{1 n}$ & $X_{1}$ \\
Agent 2 Expenditure & $Z_{21}$ & $Z_{22}$ & $\ldots$ & $Z_{2 n}$ & $X_{2}$ \\
$\ldots$ & $\ldots$ & $\ldots$ & $\ldots$ & $\ldots$ & $\ldots$ \\
Agent $n$ Expenditure & $Z_{n 1}$ & $Z_{n 2}$ & $\ldots$ & $Z_{n n}$ & $X_{n}$ \\
Sum & $Y_{1}$ & $Y_{2}$ & $\ldots$ & $Y_{n}$ & \\
\hline
\end{tabular}

\footnotetext{
${ }^{2}$ Cf. Newcomb [1] pp. 320-328. Although Irving Fisher regarded Newcomb as a pioneer of an algebraic statement of the equation of exchange (Cf. Fisher [2] p. 25), it was known from the olden days. As far as the author knows, the first writer who grasped the concept of the velocity of money was William Petty, who was a British economist in the seventeenth century (Cf. Deane [3] p. 67, Roncaglia [4] p. 390). Moreover, according to Reghinos Theocharis, the first writer who used an algebraic statement of the equation of exchange was Claus Kröncke, who was a German economist in the early nineteenth century. Joseph Lang in Germany and Samuel Turner in Britain also seem to have used the equation of exchange before Newcomb (Cf. Theocharis [5] pp. 102-110, pp. 120-121, Humphrey [6] pp. 14-16).

${ }^{3}$ It is permitted that one element is either one agent or one group of agents, but we call it merely an agent.

${ }^{4}$ We regard measures with the same quantitative units as an equivalent condition for moneys being the same kind.
} 
We call this the transfer table. From this table, we obtain

$$
Y_{1}+Y_{2}+\cdots+Y_{n}=X_{1}+X_{2}+\cdots+X_{n}
$$

That is, the sum of revenues and the sum of expenditures in the relevant space-time are equal. We call this proposition the law of transfer equality. This law reflects the fact that revenue and expenditure are the same entity from different viewpoints. That is, the revenue of somebody is the expenditure of somebody, and the expenditure of somebody is the revenue of somebody.

Quantitatively, separate transfer is expressed as a positive quantity when it is transferred, and expressed as zero when it is not transferred. Negative transfer does not have an economic meaning. Considering this with Equations (1) and (2), we see that

$$
X_{i} \geq 0, Y_{j} \geq 0, Z_{i j} \geq 0 \text { for } \forall i, j \in N
$$

always holds.

However, we suppose that expenditure in the relevant space-time is positive. The reason for this supposition is to avoid complications of the following description. If we take a measurable length as the relevant term, most agents must expend a little money. Hence, this is a reasonable supposition in practice. Using symbols, the supposition is denoted as

$$
X_{i}>0 \text { for } \forall i \in N
$$

Then, we consider the sources of money possession in the relevant space-time. Revenue is one source, but the possession at term beginning, production and being transferred from the outside of the relevant society are other sources. We decide that the sources excluding revenue are collectively called the beginning money.

On the other hand, we consider the results of money possession in the relevant space-time. Expenditure is one result, but the possession at term end, disappearance and transferring to the outside of the relevant society are other results. We decide that the results excluding expenditure are collectively called the end money.

Let $\Psi_{k}$ be the quantity of the beginning money of Agent $k$, and $\Omega_{k}$ be the quantity of the end money of Agent $k$. Quantitatively, the existence of money is expressed as a positive, and the absence of money expressed as zero. Negative existence does not have an economic meaning. ${ }^{5}$ Thus,

$$
\Psi_{k} \geq 0, \Omega_{k} \geq 0 \text { for } \forall k \in N
$$

always holds.

Then, we define the disposal as a process from the source to the result.

If we trace the source of money expended in the relevant space-time, it is only either received as revenue or included in the beginning money. We call this the disposal comprehensibility principle.

We define a disposal from revenue to expenditure as a circular disposal. We denote the circular disposal quantity of Agent $k$ as $\rho_{k}$. Moreover, let $\alpha_{k}$ be the quantity of money disposed from the beginning money to expenditure. Quantitatively, the disposal comprehensibility principle means

$$
X_{k} \leq \rho_{k}+\alpha_{k} \text { for } \forall k \in N
$$

Furthermore, money which is once received as revenue from someone is neither received from anyone else nor included in the beginning money. That is, the sources of money possession do not duplicate. We call this the disposal exclusivity principle. Quantitatively, the disposal exclusivity principle means

$$
X_{k} \geq \rho_{k}+\alpha_{k} \quad \text { for } \forall k \in N
$$

Considering both the disposal comprehensibility principle and the disposal exclusivity principle,

$$
X_{k}=\rho_{k}+\alpha_{k} \quad \text { for } \forall k \in N
$$

is satisfied.

If we trace the source of the end money, it is only either received as revenue or included in the beginning money. Let $\beta_{k}$ be the quantity of money disposed from revenue to the end money, and $\gamma_{k}$ be the quantity of

\footnotetext{
${ }^{5}$ Some economists treat debt as a negative existence of money. However, debt is only a psychological fact which is a promise to pay it back in the future. It is not a fact which is based on an objective entity of money. Whether an agent has some debt or not does not directly have an effect to the existence of money. Hence, there is not a negative existence of money. Financial relations are treated as a kind of transfer. If a loan is executed in the relevant space-time, the expenditure of the creditor and the revenue of the debtor are recorded. If a return is executed in the relevant space-time, the expenditure of the debtor and the revenue of the creditor are recorded.
} 
money disposed from the beginning money to the end money. As in the case of expenditure, considering both the disposal comprehensibility and exclusivity principle,

$$
\Omega_{k}=\beta_{k}+\gamma_{k} \quad \text { for } \forall k \in N
$$

is satisfied.

On the other hand, if we trace the result of the beginning money, it is only either expended or included in the end money. $\alpha_{k}$ refers to the quantity of money disposed from the beginning money to expenditure, and $\gamma_{k}$ refers to the quantity of money disposed from the beginning money to the end money. Therefore, considering both the disposal comprehensibility and exclusivity principle,

$$
\Psi_{k}=\alpha_{k}+\gamma_{k} \text { for } \forall k \in N
$$

is satisfied.

If we trace the result of money received as revenue in the relevant space-time, it is only either expended or included in the end money. $\rho_{k}$ refers to the quantity of money disposed from revenue to expenditure, and $\beta_{k}$ refers to the quantity of money disposed from revenue to the end money. Therefore, considering both the disposal comprehensibility and exclusivity principle,

$$
Y_{k}=\rho_{k}+\beta_{k} \quad \text { for } \forall k \in N
$$

is satisfied.

We decide that $\rho_{k}, \alpha_{k}, \beta_{k}$ and $\gamma_{k}$ are collectively called the disposal quantities. Based on their economic meanings,

$$
\rho_{k} \geq 0, \alpha_{k} \geq 0, \beta_{k} \geq 0, \gamma_{k} \geq 0 \text { for } \forall k \in N
$$

\begin{tabular}{|c|c|c|c|}
\hline Result & Expenditure & End Money & Sum \\
\hline Beginning Money & $\alpha_{k}$ & $\gamma_{k}$ & $\Psi_{k}$ \\
\hline Revenue & $\rho_{k}$ & $\beta_{k}$ & $Y_{k}$ \\
\hline Sum & $X_{k}$ & $\Omega_{k}$ & \\
\hline
\end{tabular}

is always satisfied.

The relationships between the disposal quantities and others are summarized in the following table.

We call this the disposal table of Agent $k$. Based on this table, it is obvious that

$$
X_{k}+\Omega_{k}=\Psi_{k}+Y_{k} \text { for } \forall k \in N
$$

is satisfied. That is, the gross quantity of sources of Agent $k$ and the gross quantity of results of Agent $k$ are equal. We call this proposition the law of gross disposal of Agent $k$.

If we sum Equation (8) for the whole society,

$$
\sum_{k \in N} \Psi_{k}+\sum_{k \in N} Y_{k}=\sum_{k \in N} X_{k}+\sum_{k \in N} \Omega_{k}
$$

is derived. Since $\sum_{k \in N} Y_{k}=\sum_{k \in N} X_{k}$ holds due to the law of transfer equality (Equation (3)),

$$
\Psi_{1}+\Psi_{2}+\cdots+\Psi_{n}=\Omega_{1}+\Omega_{2}+\cdots+\Omega_{n}
$$

is derived. That is, the sum of the beginning money and that of the end money are equal. We call this proposition the law of money conservation. The sum of the beginning money and that of the end money both seem to denote the abundance of money in the relevant space-time. Accordingly, the law of money conservation reflects the fact that transfer does not change the abundance of money in the whole society.

Moreover, let $F$ be a subset of $N$ excluding the empty set, and $G$ be the complement of $F$ if $F$ is a proper subset. Adding up the law of gross disposal of all agents who belong to $F$ and considering Equations (1) and (2),

$$
\sum_{k \in F} \Psi_{k}+\sum_{i \in G, k \in F} Z_{i k}+\sum_{i \in F, k \in F} Z_{i k}=\sum_{k \in F, j \in F} Z_{k j}+\sum_{k \in F, j \in G} Z_{k j}+\sum_{k \in F} \Omega_{k}
$$

is derived. Since $\sum_{i \in F, k \in F} Z_{i k}$ and $\sum_{k \in F, j \in F} Z_{k j}$ have the same contents, they are naturally equal. If we sub- 
tract $\sum_{i \in F, k \in F} Z_{i k}=\sum_{k \in F, j \in F} Z_{k j}$ from both sides of the preceding equation,

$$
\sum_{k \in F} \Psi_{k}+\sum_{i \in G, k \in F} Z_{i k}=\sum_{k \in F, j \in G} Z_{k j}+\sum_{k \in F} \Omega_{k} \quad \text { for } \forall F \subseteq N
$$

is derived. This proposition means that the sum of money which enters from the outside of $F$ is equal to that of money which exits to the outside of $F$. We call this the law of openness equality of Set $F$.

The law of money conservation (Equation (9)) is nothing but the law of openness equality of Set $N$.

\section{Formalization of the Money Circulation Equation}

Thinking normally, any money expended, received as revenue and included in the end money seems to come from the beginning money, which consists of the possession at term beginning, production and transferred from the outside. If we convert this qualitative recognition into a quantitative one, we should be able to calculate the quantity of expenditure, revenue and the end money from the quantity of the beginning money. We will look for such a method.

First, we confirm that the gross quantity of results of Agent $k$ satisfies

$$
X_{k}+\Omega_{k}>0 \text { for } \forall k \in N
$$

by Equations (5) and (6). Based on this, the expenditure rate of Agent $k$ is defined as

$$
\theta_{k}=X_{k} /\left(X_{k}+\Omega_{k}\right) \text { for } \forall k \in N
$$

In short, the expenditure rate refers to the percentage of expenditure in the gross quantity of results.

By Equations (5), (6) and (11), the expenditure rate is limited to

$$
0<\theta_{k} \leq 1 \text { for } \forall k \in N
$$

Considering Equations (2), (8) and (12), we can derive

$$
X_{k}=\theta_{k}\left(\Psi_{k}+Z_{1 k}+Z_{2 k}+\cdots+Z_{n k}\right) \text { for } \forall k \in N
$$

Next, based on $X_{i}>0$ (Equation (5)), the expenditure distribution rate from Agent $i$ to Agent $j$ is defined as

$$
\mu_{i k}=Z_{i k} / X_{i} \text { for } \forall i, k \in N
$$

In short, the expenditure distribution rate refers to a percentage of the separate transfer in expenditure.

By Equations (1), (4) and (5), the range of the expenditure distribution rate is limited to

$$
0 \leq \mu_{i k} \leq 1 \text { for } \forall i, k \in N
$$

Moreover, considering Equations (1) and (15), we can see that

$$
\mu_{i 1}+\mu_{i 2}+\cdots+\mu_{i n}=1 \text { for } \forall i \in N
$$

Considering Equations (14) and (15), we can derive

$$
\left(1-\theta_{k} \mu_{k k}\right) X_{k}-\sum_{h \neq k} \theta_{k} \mu_{h k} X_{h}=\theta_{k} \Psi_{k} \quad \text { for } \forall k \in N
$$

We collect this formula from $k=1$ to $n$ and regard them as a system of simultaneous equations in $X_{1}, X_{2}, \cdots, X_{n}$. It is the money circulation equation.

Let $E$ be a unit matrix, and $x$ be a column vector with the ith element equal to $X_{i}$, and $\psi$ be a column vector with the $h$ th element equal to $\Psi_{h}$, and $\Theta$ be a diagonal square matrix with the $h$ th diagonal element equal to $\theta_{h}$, and $\mu$ be a square matrix with the $(i, j)$ th element equal to $\mu_{j i}$. Then, the money circulation equation can be denoted as

$$
(E-\Theta \mu) x=\Theta \psi
$$

Moreover, let $p_{i h}$ be the $(i, h)$ th element of the inverse of $E-\Theta \mu$. The unique solution of the money circulation equation can be expressed as

$$
X_{i}=\sum_{h \in N} p_{i h} \theta_{h} \Psi_{h} \quad \text { for } \forall i \in N
$$


$p_{i h}$ can be calculated from the expenditure rate and the distribution rate of all agents. Therefore, if the money circulation equation has a unique solution, each expenditure quantity can be calculated from the expenditure rate, the distribution rate and the beginning money.

Note that, $\Theta \mu$ is a non-negative matrix because $\theta_{i} \mu_{j i} \geq 0$ from Equations (13) and (16). Hence, $E-\Theta \mu$ is a Z-matrix, which is defined as a square matrix in which the non-diagonal elements are all non-positive. Moreover, we can obtain $\Theta \psi \geq 0$ from Equations (6) and (13). Considering this with Equation (5), we find that $(E-\Theta \mu) x \geq 0$ where $\exists x>0$ is satisfied. If this type of matrix is non-singular, $p_{i h}$, which is an element of the inverse of $E-\Theta \mu$, has the following peculiarities. ${ }^{6}$

$p_{i h}$ is non-negative generally. Considering this with Equation (13), an increase of the beginning money does not decrease expenditure unless it causes the variation of the expenditure rate or the distribution rate.

In particular, $p_{i i}$ is 1 or more. Considering this with Equation (13), if one unit of the beginning money of a certain agent increases, their own expenditure increases equal to the expenditure rate or more unless it causes a variation of the expenditure rate or the distribution rate.

Now, using Equations (15) and (18),

$$
Z_{i j}=\mu_{i j}\left(\sum_{h \in N} p_{i h} \theta_{h} \Psi_{h}\right) \text { for } \forall i, j \in N
$$

is derived. Each separate transfer quantity can be also calculated from the expenditure rate, the distribution rate and the beginning money.

Moreover, due to Equations (2) and (19),

$$
Y_{j}=\sum_{i \in N} \mu_{i j}\left(\sum_{h \in N} p_{i h} \theta_{h} \Psi_{h}\right) \text { for } \forall j \in N
$$

is derived. Each revenue quantity can also be calculated from the expenditure rate, the distribution rate and the beginning money.

Furthermore, because of Equations (8), (18) and (20),

$$
\Omega_{k}=\Psi_{k}+\sum_{i \in N} \mu_{i k}\left(\sum_{h \in N} p_{i h} \theta_{h} \Psi_{h}\right)-\sum_{h \in N} p_{k h} \theta_{h} \Psi_{h} \quad \text { for } \forall k \in N
$$

is derived. Each end money quantity can also be calculated from the expenditure rate, the distribution rate and the beginning money.

Thus, if the money circulation equation has a unique solution, we can calculate all of the quantities of expenditure, separate transfer, revenue and the end money from the quantity of the beginning money with the help of the expenditure rate and the expenditure distribution rate.

\section{Solvability Condition of the Money Circulation Equation}

If the money circulation equation has a unique solution, the amount of money which is expended, received as revenue, and included in the end money can be calculated. However, if it does not have a unique solution, we cannot execute such calculations naturally. The effectiveness of the money circulation equation depends on the solvability of the equation. Hence, we will look for a solvability condition for it.

As we confirmed in the preceding section, the money circulation equation is denoted as $(E-\Theta \mu) x=\Theta \psi$. This set of simultaneous equations has a unique solution if and only if $E-\Theta \mu$ is non-singular.

We also confirmed in the preceding section that $E-\Theta \mu$ is a Z-matrix which satisfies $(E-\Theta \mu) x \geq 0$ where $\exists x>0$. There exists the following necessary and sufficient condition for non-singularity of $E-\Theta \mu$.

Let $F$ be a subset of the relevant society $N$ which is not empty and $G$ be the complement of $F$ if $F$ is a proper subset. The condition is $\exists k \in F$ such that $\theta_{k} \Psi_{k}>0$ or $\not \mathcal{A} \not \mathcal{G}, \exists k \in F$ such that $-\theta_{k} \mu_{i k}<0$ for $\forall F \subseteq N$. Considering Equations (5), (13) and (15), a solvability condition can be rewritten as follows.

Theorem 3.1. Let $F$ be a subset of the relevant society $N$ which is not empty and $G$ be the complement of $F$ if $F$ is a proper subset of $N$. The money circulation equation has a unique solution if and only if $\exists k \in F$ such that $\Psi_{k}>0$ or $\exists i \in G, \quad \exists k \in F$ such that $Z_{i k}>0$ for $\forall F \subseteq N .{ }^{8}$

Moreover, there exists another solvability condition. In order to show it, we prove the following theorem.

${ }^{6} \mathrm{Cf}$. Theorems 1.4 and 3.6 in Miura [7]. $E-\Theta \mu$ is an NPZ-matrix called in Miura [7].

${ }^{7} \mathrm{Cf}$. Theorem 3.12 in Miura [7]. The prototype of this condition was first shown in Beauwens [8] and Neumann [9].

${ }^{8}$ In the case $F=N$, this condition is merely $\exists k \in F$ such that $\Psi_{k}>0$. 
Theorem 3.2. $\exists k \in F$ such that $\Psi_{\mathrm{k}}>0$ or $\exists i \in G, \exists k \in F$ such that $Z_{i k}>0$ for $\forall F \subseteq N$ is equivalent to $\exists k \in F$ such that $\Omega_{k}>0$ or $\exists k \in F, \exists j \in G$ such that $Z_{k j}>0$ for $\forall F \subseteq N$.

Proof. Considering $\Psi_{k} \geq 0$ (Equation (6)) and $Z_{i k} \geq 0$ (Equation (4)), $\exists k \in F$ such that $\Psi_{k}>0$ or $\exists i \in G$, $\exists k \in F$ such that $Z_{i k}>0$ if and only if $\sum_{k \in F} \Psi_{k}+\sum_{i \in G, k \in F} Z_{i k}>0$. By the law of openness equality of Set $F$ (Equation (10)), this is equivalent to $\sum_{k \in F, j \in G} Z_{k j}+\sum_{k \in F} \Omega_{k}>0$. Considering $\Omega_{k} \geq 0$ (Equation (6)) and $Z_{k j} \geq 0$ (Equation (4)), this is equivalent to $\exists k \in F$ such that $\Omega_{k}>0$ or $\exists k \in F, \exists j \in G$ such that $\mathrm{Z}_{k j}>0$. [Q. E. D.] From Theorems 3.1 and 3.2, the following solvability condition of the money circulation equation is derived.

Theorem 3.3. Let $F$ be a subset of the relevant society $N$ which is not empty and $G$ be the complement of $F$ if $F$ is a proper subset of $N$. The money circulation equation has a unique solution if and only if $\exists k \in F$ such that $\Omega_{k}>0$ or $\exists k \in F, \exists j \in G$ such that $Z_{k j}>0$ for $\forall F \subseteq N .{ }^{9}$

We will interpret two solvability conditions shown in Theorems 3.1 and 3.3.

" $\exists k \in F$ such that $\Psi_{k}>0$ " expresses the fact that money enters a part of the relevant society from the outside of the relevant space-time. Moreover, " $\exists i \in G, \exists k \in F$ such that $Z_{i k}>0$ ” expresses the fact that money enters there from the space-time which is the outside of the part but the inside of the relevant space-time. Thus, the solvability condition in Theorem 3.1 refers to the fact that money in each part of the relevant society enters from its spatio-temporal outside.

On the other hand, " $\exists k \in F$ such that $\Omega_{k}>0$ " expresses the fact that money exits a part of the relevant society to the outside of the relevant space-time. Moreover, " $\exists k \in F, \exists j \in G$ such that $Z_{k j}>0$ " expresses the fact that money exits there to the space-time which is the outside of the part but the inside of the relevant space-time. Thus, a solvability condition in Theorem 3.3 refers to the fact that money in each part of the relevant society exits to its spatio-temporal outside.

Both conditions express the fact that each part of the relevant society is open to its outside. The former expresses the openness for the source direction and the latter for the result direction. Accordingly, Theorems 3.1 and 3.3 insist that the money circulation equation has a unique solution if and only if each part of the relevant society is open to its outside. We call these conditions the space-time openness conditions of the money circulation structure. These conditions seem to be relevant to the bounded confidence condition in the literature of social opinion evolution. ${ }^{10}$

Then, is the openness condition always true? Directly, it is not self-evident. However, the space-time openness is always true under an ordinary premise about time. In order to clarify this, we will examine the temporal structure of a monetary economy.

We divide the relevant term into several subterms. Dividing it into $t$ subterms, we denote the relevant term by $T=\{1,2, \cdots, t\}$. However, we set the index of subterms such that a smaller index corresponds to an earlier subterm and a larger index corresponds to a later subterm.

Let $X_{i(u)}$ be the expenditure quantity of Agent $i$ in Subterm $u$, and $Y_{j(u)}$ be the revenue quantity of Agent $j$ in Subterm $u$, and $Z_{i j(u)}$ be the separate transfer quantity from Agent $i$ to Agent $j$ in Subterm $u$.

The next relations exist between the whole quantities in the relevant term and separate quantities in each subterm.

$$
\begin{array}{cc}
X_{i}=X_{i(1)}+X_{i(2)}+\cdots+X_{i(t)} & \text { for } \forall i \in N \\
Y_{j}=Y_{j(1)}+Y_{j(2)}+\cdots+Y_{j(t)} & \text { for } \forall j \in N \\
Z_{i j}=Z_{i j(1)}+Z_{i j(2)}+\cdots+Z_{i j(t)} & \text { for } \forall i, j \in N
\end{array}
$$

Expenditure in Subterm $u$ is defined as transferring money within Subterm $u$. Moreover, revenue in Subterm $u$ is defined as money being transferred into Subterm $u$. Therefore, the next relations are satisfied in Subterm $u$.

$$
\begin{array}{ll}
Z_{i 1(u)}+Z_{i 2(u)}+\cdots+Z_{i n(u)}=X_{i(u)} & \text { for } \forall i \in N, \forall u \in T \\
Z_{1 j(u)}+Z_{2 j(u)}+\cdots+Z_{n j(u)}=Y_{j(u)} & \text { for } \forall j \in N, \forall u \in T
\end{array}
$$

The transfer table in Subterm $u$ is as follows.

\footnotetext{
${ }^{9}$ In the case $F=N$, this condition is merely $\exists k \in F$ such that $\Omega_{k}>0$.

${ }^{10}$ The recent works of this topic are Varshney [10], Shang [11], Shang [12].
} 


\begin{tabular}{ccclcc}
\hline & Agent 1 Revenue & Agent 2 Revenue & $\ldots$ & Agent $n$ Revenue & Sum \\
\hline Agent 1 Expenditure & $Z_{11(u)}$ & $Z_{12(u)}$ & $\ldots$ & $Z_{1 n(u)}$ & $X_{1(u)}$ \\
Agent 2 Expenditure & $Z_{21(u)}$ & $Z_{22(u)}$ & $\ldots$ & $Z_{2 n(u)}$ & $X_{2(u)}$ \\
$\ldots$ & $\ldots$ & $\ldots$ & $\ldots$ & $\ldots$ & $\ldots$ \\
Agent $n$ Expenditure & $Z_{n 1(u)}$ & $Z_{n 2(u)}$ & $\ldots$ & $Z_{n n(u)}$ & $X_{n(u)}$ \\
Sum & $Y_{1(u)}$ & $Y_{2(u)}$ & $\ldots$ & $Y_{n(u)}$ & \\
\hline
\end{tabular}

From this transfer table, we obtain

$$
Y_{1(u)}+Y_{2(u)}+\cdots+Y_{n(u)}=X_{1(u)}+X_{2(u)}+\cdots+X_{n(u)} \quad \text { for } \forall u \in T
$$

That is, the sum of revenues and the sum of expenditures in each subterm are equal. Although we have already obtained the law of transfer equality in the relevant term (Equation (3)), the same law is also satisfied in each subterm.

Further, as per Equation (4), each expenditure, revenue and separate transfer in each subterm is non-negative by their economic meaning.

$$
X_{i(u)} \geq 0, Y_{j(u)} \geq 0, Z_{i j(u)} \geq 0 \text { for } \forall i, j \in N, \forall u \in T
$$

Then, let $\rho_{k(p q)}$ be the circular disposal quantity which Agent $k$ disposed from revenue in Subterm $p$ to expenditure in Subterm $q$. Let $\alpha_{k(q)}$ be the disposal quantity which Agent $k$ disposed from the beginning money to expenditure in Subterm $q$. Let $\beta_{k(p)}$ be the quantity which Agent $k$ disposed from revenue in Subterm $p$ to the end money.

As per Equation (7), they are all non-negative.

$$
\rho_{k(p q)} \geq 0, \alpha_{k(q)} \geq 0, \beta_{k(p)} \geq 0 \quad \text { for } \forall k \in N, \forall p, q \in T
$$

The disposal table of Agent $k$ considering term division is as follows.

\begin{tabular}{ccccccc}
\hline Result & $\begin{array}{c}\text { Subterm } 1 \\
\text { Expenditure }\end{array}$ & $\begin{array}{c}\text { Subterm } 2 \\
\text { Expenditure }\end{array}$ & $\ldots$ & $\begin{array}{c}\text { Subterm } t \\
\text { Expenditure }\end{array}$ & End Money & Sum \\
\hline Source & $\alpha_{k(1)}$ & $\alpha_{k(2)}$ & $\ldots$ & $\alpha_{k(t)}$ & $\gamma_{k}$ & $\Psi_{k}$ \\
Beginning Money & $\rho_{k(1)}$ & $\rho_{k(12)}$ & $\ldots$ & $\rho_{k(1)}$ & $\beta_{k(1)}$ & $Y_{k(1)}$ \\
Subterm 1 Revenue & $\rho_{k(21)}$ & $\rho_{k(22)}$ & $\ldots$ & $\rho_{k(2 t)}$ & $\beta_{k(2)}$ & $Y_{k(2)}$ \\
Subterm 2 Revenue & $\ldots$ & $\ldots$ & $\ldots$ & $\ldots$ & $\ldots$ & $\ldots$ \\
$\ldots$ & $\rho_{k(1)}$ & $\rho_{k(2)}$ & $\ldots$ & $\rho_{k(t)}$ & $\beta_{k(t)}$ & $Y_{k(t)}$ \\
Subterm $t$ Revenue & $X_{k(1)}$ & $X_{k(2)}$ & $\ldots$ & $X_{k(t)}$ & $\Omega_{k}$ & \\
\hline Sum & & & & & &
\end{tabular}

Note that, based on our common sense about time, beings in the future cannot travel into the past. Moreover, it is usually thought that the same being cannot exist in different places at exactly the same time. Considering these, beings can move only from the past to the future.

If we apply a similar principle to money transfer, money cannot be disposed from a future revenue to a past expenditure. Moreover, money received at a certain time cannot be expended at exactly the same time. In other words, money can be disposed from revenue of the past to expenditure of the future. We call this the disposal irreversibility principle.

We now think about the relationship between the disposal irreversibility principle and circular disposal quantities. Based on the disposal irreversibility principle, circular quantities from the future to the past are naturally zeroes. Then, we think about the sign of simultaneous circular quantities. Based on Equation (23), it is always non-negative. However, we cannot judge whether it is positive or zero generally. For example, if subterms are divided monthly, revenue in a certain month may be or may not be expended in the same month. If they are divided weekly or daily, a similar situation occurs.

However, the revenue time and the expenditure time of one disposal are not exactly the same time by the disposal irreversibility principle. Hence, we can divide the relevant term between both times. Then, they belong to 
different subterms. If the number of disposals in the relevant space-time is finite $m,{ }^{11}$ each revenue time and expenditure time of all disposals belong to different subterms by at most a finite $m$ times division. If we define a subterm whose simultaneous circular quantities are zeroes for any agent as a basic-term, this fact means that the relevant term can always be divided into finite basic-terms.

These conclusions are summarized as follows.

Theorem 3.4. Under the premise of the disposal irreversibility principle, $\rho_{k(p q)}=0$ holds for $\forall k \in N$, $\forall p>q$. Furthermore, the relevant term can always be divided into a finite number of basic-terms where a basic-term refers to a Subterm $u$ such that $\rho_{k(u u)}=0$ for $\forall k \in N$.

Based on Theorem 3.4, we can prove that the space-time openness condition is guaranteed by the disposal irreversibility principle.

Theorem 3.5. Under the premise of the disposal irreversibility principle, $\exists k \in F$ such that $\Psi_{k}>0$ or $\exists i \in G, \exists k \in F$ such that $Z_{i k}>0$ for $\forall F \subseteq N$.

Proof. It is enough to prove $\exists k \in F$ such that $\Psi_{k}>0$ if $Z_{i j}=0$ for $\forall i \in G, \forall j \in F$. Based on Theorem 3.4, we divide the relevant term into finite basic-terms. Due to Equation (5), all agents expend in some basic-terms. Let Agent $k \in F$ be the agent who expends first in $F$. Let Subterm $u$ be a basic-term when Agent $k$ expends first. $^{12}$

First, we will prove the case $u$ is not the first or the last basic-term.

Let Subterm - be the set of basic-terms which are before Subterm $u$, and Subterm+ be the set of basic-terms which are after Subterm $u$. In this case, the disposal table of Agent $k$ is as follows.

\begin{tabular}{cccccc}
\hline Result & $\begin{array}{c}\text { Subterm- } \\
\text { Expenditure }\end{array}$ & $\begin{array}{c}\text { Subterm } u \\
\text { Expenditure }\end{array}$ & $\begin{array}{c}\text { Subterm+ } \\
\text { Expenditure }\end{array}$ & $\begin{array}{c}\text { End } \\
\text { Money }\end{array}$ & Sum \\
\hline Source & $\alpha_{k(-)}$ & $\alpha_{k(u)}$ & $\alpha_{k(+)}$ & $\gamma_{k}$ & $\Psi_{k}$ \\
Beginning Money & $\rho_{k(-)}$ & $\rho_{k(-u)}$ & $\rho_{k(+)}$ & $\beta_{k(-)}$ & $Y_{k(-)}$ \\
Subterm- Revenue & $\rho_{k(u-)}$ & $\rho_{k(u u)}$ & $\rho_{k(u+)}$ & $\beta_{k(u)}$ & $Y_{k(u)}$ \\
Subterm $u$ Revenue & $\rho_{k(+)}$ & $\rho_{k(+u)}$ & $\rho_{k(++)}$ & $\beta_{k(+)}$ & $Y_{k(+)}$ \\
Subterm+ Revenue & $X_{k(-)}$ & $X_{k(u)}$ & $X_{k(+)}$ & $\Omega_{k}$ & \\
Sum & & & & \\
\hline
\end{tabular}

By Theorem 3.4, $\rho_{k(u-)}=0, \rho_{k(+-)}=0$ and $\rho_{k(+u)}=0$ are satisfied. Moreover, $\rho_{k(u u)}=0$ is also satisfied because Subterm $u$ is a basic-term, and $X_{k(u)}>0$ because $u$ is defined as the first basic-term whose expenditure of $k$ is positive. We put these facts in the disposal table.

\begin{tabular}{cccccc}
\hline Result & $\begin{array}{c}\text { Subterm- } \\
\text { Expenditure }\end{array}$ & $\begin{array}{c}\text { Subterm } u \\
\text { Expenditure }\end{array}$ & $\begin{array}{c}\text { Subterm+ } \\
\text { Expenditure }\end{array}$ & $\begin{array}{c}\text { End } \\
\text { Money }\end{array}$ & Sum \\
\hline Bource & $\alpha_{k(-)}$ & $\alpha_{k(u)}$ & $\alpha_{k(+)}$ & $\gamma_{k}$ & $\Psi_{k}$ \\
Subterm - Revenue & $\rho_{k(-)}$ & $\rho_{k(-u)}$ & $\rho_{k(+)}$ & $\beta_{k(-)}$ & $Y_{k(-)}$ \\
Subterm $u$ Revenue & 0 & 0 & $\rho_{k(u+)}$ & $\beta_{k(u)}$ & $Y_{k(u)}$ \\
Subterm+ Revenue & 0 & 0 & $\rho_{k(++)}$ & $\beta_{k(+)}$ & $Y_{k(+)}$ \\
Sum & $X_{k(-)}$ & + & $X_{k(+)}$ & $\Omega_{k}$ & \\
\hline
\end{tabular}

Next, we will prove $Y_{k(-)}=0$ by dividing it into two cases.

1) The case $F=N$.

Subterm $u$ is the first basic-term whose expenditure in $F=N$ is positive and Subterm- is before $u$. Thus, $\forall X_{i(-)}=0$. Hence, $\sum_{j \in N} Y_{j(-)}=0$ from the law of transfer equality (Equation (21)). Considering Equation (22), $\forall Y_{j(-)}=0$ is derived. Therefore, $Y_{k(-)}=0$.

2) The case $F \subsetneq N$.

We write the transfer table of Subterm-divided into $F$ and $G$.

\footnotetext{
${ }^{11}$ Since the finiteness of the relevant term is assumed, the number of disposals which can be justified empirically is only a finite number.

${ }^{12} \mathrm{As}$ the relevant term can be divided into finite basic-terms, the existence of the first basic-term is always guaranteed.
} 


\begin{tabular}{cccc}
\hline & Set $F$ Revenue & Set $G$ Revenue & Sum \\
\hline Set $F$ Expenditure & $\sum_{i \in F, j \in F} Z_{i j(-)}$ & $\sum_{i \in F, j \in G} Z_{i j(-)}$ & $\sum_{i \in F} X_{i(-)}$ \\
Set $G$ Expenditure & $\sum_{i \in G, j \in F} Z_{i j(-)}$ & $\sum_{i \in G, j \in G} Z_{i j(-)}$ & $\sum_{i \in G} X_{i(-)}$ \\
Sum & $\sum_{j \in F} Y_{j(-)}$ & $\sum_{j \in G} Y_{j(-)}$ & \\
\hline
\end{tabular}

Subterm $u$ is the first basic-term whose expenditure in $F$ is positive and Subterm- is before $u$. Hence, $\sum_{i \in F} X_{i(-)}=0$. Moreover, $\sum_{i \in G, j \in F} Z_{i j(-)}=0$ from the premise $Z_{i j}=0$ for $\forall i \in G, \forall j \in F$. We put these facts in the preceding transfer table.

\begin{tabular}{cccc}
\hline & Set $F$ Revenue & Set $G$ Revenue & Sum \\
\hline Set $F$ Expenditure & $\sum_{i \in F, j \in F} Z_{i j(-)}$ & $\sum_{i \in F, j \in G} Z_{i j(-)}$ & 0 \\
Set $G$ Expenditure & 0 & $\sum_{i \in G, j \in G} Z_{i j(-)}$ & $\sum_{i \in G} X_{i(-)}$ \\
Sum & $\sum_{j \in F} Y_{j(-)}$ & $\sum_{j \in G} Y_{j(-)}$ & \\
\hline
\end{tabular}

Here, we focus on the row of Set F. Since all separate transfers are non-negative (Equation (22)), the elements of this row are all zeroes. We put these facts in the preceding transfer table.

\begin{tabular}{cccc}
\hline & Set $F$ Revenue & Set $G$ Revenue & Sum \\
\hline Set $F$ Expenditure & 0 & 0 & 0 \\
Set $G$ Expenditure & 0 & $\sum_{i \in G, j \in G} Z_{i j(-)}$ & $\sum_{i \in G} X_{i(-)}$ \\
Sum & $\sum_{j \in F} Y_{j(-)}$ & $\sum_{j \in G} G_{j(-)}$ & \\
\hline
\end{tabular}

Next, we focus on the column of Set $F$. We can see that $\sum_{j \in F} Y_{j(-)}=0$. As $k$ belongs to $F, Y_{k(-)}=0$ is derived.

Now, we have confirmed that $Y_{k(-)}=0$ is satisfied generally. We add this fact to the preceding disposal table of $k$ to obtain the following.

\begin{tabular}{cccccc}
\hline Result & $\begin{array}{c}\text { Subterm- } \\
\text { Expenditure }\end{array}$ & $\begin{array}{c}\text { Subterm } u \\
\text { Expenditure }\end{array}$ & $\begin{array}{c}\text { Subterm+ } \\
\text { Expenditure }\end{array}$ & $\begin{array}{c}\text { End } \\
\text { Money }\end{array}$ & Sum \\
\hline Source & $\alpha_{k(-)}$ & $\alpha_{k(u)}$ & $\alpha_{k(+)}$ & $\gamma_{k}$ & $\Psi_{k}$ \\
Beginning Money & $\rho_{k(-)}$ & $\rho_{k(-u)}$ & $\rho_{k(+)}$ & $\beta_{k(-)}$ & 0 \\
Subterm- Revenue & 0 & 0 & $\rho_{k(u+)}$ & $\beta_{k(u)}$ & $Y_{k(u)}$ \\
Subterm $u$ Revenue & 0 & 0 & $\rho_{k(++)}$ & $\beta_{k(+)}$ & $Y_{k(+)}$ \\
Subterm+ Revenue & $X_{k(-)}$ & + & $X_{k(+)}$ & $\Omega_{k}$ & \\
Sum & & & & \\
\hline
\end{tabular}

Now, we focus on the row of Subterm-. Since all disposal quantities are non-negative by Equation (23), all elements of this row are zeroes. We add these facts to the preceding disposal table and obtain the following.

\begin{tabular}{cccccc}
\hline Result & $\begin{array}{c}\text { Subterm- } \\
\text { Expenditure }\end{array}$ & $\begin{array}{c}\text { Subterm } u \\
\text { Expenditure }\end{array}$ & $\begin{array}{c}\text { Subterm+ } \\
\text { Expenditure }\end{array}$ & $\begin{array}{c}\text { End } \\
\text { Money }\end{array}$ & Sum \\
\hline Source & $\alpha_{k(-)}$ & $\alpha_{k(u)}$ & $\alpha_{k(+)}$ & $\gamma_{k}$ & $\Psi_{k}$ \\
Beginning Money & 0 & 0 & 0 & 0 & 0 \\
Subterm- Revenue & 0 & 0 & $\rho_{k(u+)}$ & $\beta_{k(u)}$ & $Y_{k(u)}$ \\
Subterm $u$ Revenue & 0 & 0 & $\rho_{k(++)}$ & $\beta_{k(+)}$ & $Y_{k(+)}$ \\
Subterm+ Revenue & $X_{k(-)}$ & + & $X_{k(+)}$ & $\Omega_{k}$ & \\
\hline
\end{tabular}


Next, we focus on the column of Subterm $u$. We can see that $\alpha_{k(u)}>0$. We add this fact to the preceding disposal table and obtain the following.

\begin{tabular}{cccccc}
\hline Result & $\begin{array}{c}\text { Subterm- } \\
\text { Expenditure }\end{array}$ & $\begin{array}{c}\text { Subterm } u \\
\text { Expenditure }\end{array}$ & $\begin{array}{c}\text { Subterm+ } \\
\text { Expenditure }\end{array}$ & $\begin{array}{c}\text { End } \\
\text { Money }\end{array}$ & Sum \\
\hline Source & $\alpha_{k(-)}$ & + & $\alpha_{k(+)}$ & $\gamma_{k}$ & $\Psi_{k}$ \\
Beginning Money & 0 & 0 & 0 & 0 & 0 \\
Subterm- Revenue & 0 & 0 & $\rho_{k(u+)}$ & $\beta_{k(u)}$ & $Y_{k(u)}$ \\
Subterm $u$ Revenue & 0 & 0 & $\rho_{k(++)}$ & $\beta_{k(+)}$ & $Y_{k(+)}$ \\
Subterm+ Revenue & $X_{k(-)}$ & + & $X_{k(+)}$ & $\Omega_{k}$ & \\
\hline Sum & & & &
\end{tabular}

Then, we focus on the row of the beginning money. As all disposal quantities are non-negative, we obtain $\Psi_{k}>0$. The proof in the case $u$ is not the first or the last basic-term is complete.

In the case $u$ is the first basic-term; you should assume the row and column of Subterm- do not exist. In the case $u$ is the last basic-term; you should assume the row and column of Subterm+ do not exist. Then, imitating the previous proof, you can easily prove $\Psi_{k}>0$. [Q. E. D.]

From Theorems 3.1 and 3.5, we obtain the following theorem about the solvability of the money circulation equation.

Theorem 3.6. Under the premise of the disposal irreversibility principle, the money circulation equation has a unique solution.

If you compare this paper with Miura [13], you will notice that the proof for the solvability of the money circulation equation is similar to that of the economic input-output equation. However, the details are different.

First, each equation is formalized as the positions of the sources and the results are opposite. Thus, both proofs that the disposal irreversibility principle causes the space-time openness are also opposite each other.

Second, each economic structure which is the background of each equation has a different peculiarity about the relationship between separate quantities and the gross quantity. We can add up only regarding the result in the input-output structure, but we can add up regarding both the source and the result in the money circulation structure. Therefore, by the disposal irreversibility principle, the former structure is open only to the result, whereas the latter structure is open to both the source and the result.

However, both equations surely have same peculiarities that a time irreversible disposal guarantees their solvability. Both solvability conditions teach us the importance of considering time irreversibility when we think about economic circulations.

\section{Comparison with Augustinovics' Money Circulation Equation}

In fact, the equation which resembles the money circulation equation in this paper was already shown by Mária Augustinovics, who is a Hungarian economist. Her work may be affected by the economic input-output theory invented by Wassily Leontief. ${ }^{13}$

When she disclosed this equation, Hungary was still under a planned economy, and the central bank of Hungary had a monopolistic control of credit and money circulation. Under such a situation, while working at Hungarian Planning Office, she showed her money circulation equation in order to support an economic planning. ${ }^{14}$ This paper will introduce it. However, we use different expressions from her in part.

We decide that the relevant society consists of $n$ civilian agents who possess money. We denote the relevant society by $N=\{1,2, \cdots, n\}$. The receipt of each agent consists of real receipts from civilian agents and credits from the central bank.

Let $b_{x}$ be the gross receipt of Agent $x$, and $q_{y x}$ be the separate transfer through civilian trading from Agent $y$ to Agent $x$, and $h_{x}$ be the credit of Agent $x$ supplied from the bank. The following relation is satisfied between them.

$$
b_{x}=q_{1 x}+q_{2 x}+\cdots+q_{n x}+h_{x} \quad \text { for } \forall x \in N
$$

\footnotetext{
${ }^{13}$ In the paper that she clarified her money circulation equation, there exists a description regarding the input-output theory (Cf. Augustinovics [14] p. 45). Leontief seems to have known her equation because the paper which he wrote with András Brody refers to Augustinovics [14 (Cf. Leontief \& Brody [15] p. 225).

${ }^{14}$ Augustinovics [14] pp. 44-45.
} 
Here, we define $k_{y x}$ as

$$
k_{y x}=q_{y x} / b_{y} \text { for } \forall x, y \in N
$$

Considering Equations (24) and (25), we can derive

$$
\left(1-k_{x x}\right) b_{x}-\sum_{y \neq x} k_{y x} b_{y}=h_{x} \quad \text { for } \forall x \in N
$$

We collect this formula from $x=1$ to $n$ and regard them as a system of simultaneous equations in $b_{1}, b_{2}, \cdots, b_{n}$. It is Augustinovics' money circulation equation.

Let $E$ be a unit matrix, and $b$ be the column vector with the $y$ th element equal to $b_{y}$, and $h$ be a column vector with the $x$ th element equal to $h_{x}$, and $K$ be a square matrix with the $(x, y)$ th element equal to $k_{y x}$. Then, Augustinovics' money circulation equation can be denoted as $(E-K) b=h$.

Moreover, let $r_{y x}$ be the $(y, x)$ th element of the inverse of $E-K$. The solution of Augustinovics' equation can be denoted as $b_{y}=\sum_{x \in N} r_{y x} h_{x}$ for $\forall y \in N$. Further, considering Equation (25), we can derive $q_{y z}=k_{y z}\left(\sum_{x \in N} r_{y x} h_{x}\right)$ for $\forall y, z \in N$. Since $r_{y x}$ can be calculated from $k_{i j}$ of all civilian agents, the gross receipt of each agent and each separate transfer can be calculated from $h_{i}$ and $k_{i j}$ of all agents.

Many readers may feel that Augustinovics' equation resembles our money circulation equation. However, they do not share every point. We will examine the commonalities and differences between the two equations.

What does each concept of Augustinovics' equation correspond to in our equation?

As we confirmed before, $h_{i}$ expresses the credit supplied from the bank. If most money consists of credit, it can be interpreted that $h_{i}$ corresponds to the beginning money $\left(\Psi_{i}\right)$ in our equation.

If we interpret our transfer as restricted to the civilian trading, $q_{i j}$ corresponds to the separate transfer $\left(Z_{i j}\right)$ in our equation. Thus, $\sum_{j \in N} q_{i j}$ corresponds to expenditure $\left(X_{i}\right)$ and $\sum_{i \in N} q_{i j}$ corresponds to revenue $\left(Y_{j}\right)$. Therefore, $b_{i}$ corresponds to the gross source $\left(\Psi_{i}+Y_{i}\right)$.

The two equations are common at the point to be calculated from the beginning money, but they differ at the point that Augustinovics' equation calculates the gross source where our equation calculates expenditure.

Then, what does $k_{i j}$, which is the $(j, i)$ th coefficient of Augustinovics' equation, correspond to? Based on Equation (25), it corresponds to $Z_{i j} /\left(\Psi_{i}+Y_{i}\right)$ directly. By the law of gross disposal (Equation (8)), it can be rewritten to $Z_{i j} /\left(X_{i}+\Omega_{i}\right)$. Further, we can transform it as follows.

$$
k_{i j}=Z_{i j} /\left(X_{i}+\Omega_{i}\right)=\left\{X_{i} /\left(X_{i}+\Omega_{i}\right)\right\}\left(Z_{i j} / X_{i}\right)=\theta_{i} \mu_{i j}
$$

That is, $k_{i j}$ corresponds to the product of the expenditure rate and the expenditure distribution rate. In other words, it is the separate expenditure rate. On the other hand, the $(j, i)$ th coefficient of our equation is $\theta_{j} \mu_{i j}$. Both coefficients are common at the point of the product of the expenditure rate and the distribution rate. However, their subscripts of the expenditure rate are different. It is appropriate that the coefficient of Augustinovics' equation is called a separate expenditure rate, but it is not appropriate that the coefficient of our equation is called like that.

The latter can be transformed to

$$
\theta_{j} \mu_{i j}=\left\{X_{j} /\left(X_{j}+\Omega_{j}\right)\right\}\left(Z_{i j} / X_{i}\right)=\left\{Z_{i j} /\left(X_{j}+\Omega_{j}\right)\right\}\left(X_{j} / X_{i}\right)
$$

$Z_{i j} /\left(X_{j}+\Omega_{j}\right)$ should not be called the separate expenditure rate because $Z_{i j}$ is not a part of $X_{j}$ but of $X_{i}$. Since $X_{j}+\Omega_{j}=\Psi_{j}+Y_{j}$ holds by the law of gross disposal (Equation (8)), $Z_{i j} /\left(X_{j}+\Omega_{j}\right)$ is equal to $Z_{i j} /\left(\Psi_{j}+Y_{j}\right)$. As $Z_{i j}$ is a part of $Y_{j}, Z_{i j} /\left(X_{j}+\Omega_{j}\right)=Z_{i j} /\left(\Psi_{j}+Y_{j}\right)$ should be called the separate revenue rate. In addition, $X_{j} / X_{i}$ is included in the above expression. Like this, the coefficient of our equation is different from that of Augustinovics' equation.

Eventually, Augustinovics' equation is a method which calculates the gross source from the beginning money and the separate expenditure rate, whereas our equation calculates expenditure from the beginning money, the expenditure rate and the expenditure distribution rate.

The two equations are not the same exactly, but they are certainly similar. Therefore, Augustinovics is qualified to be called a pioneer of our monetary theory. 


\section{Concluding Comments}

Although Augustinovics' money circulation equation resembles our money circulation equation, we have given new and distinct findings about the solvability of the equation.

It is certain that descriptions about the solvability exist in Augustinovics' paper. She said:

"If the $j$-th holder of money raises one unit of credit from the bank and spends it according to his own structure of outlays, the money thus created flows from him to the other money-holders... For the others, receipts are generated and they, too, spend them according to their own structure of outlays. The money, therefore, will be diffused again... In this manner receipts are again generated for the most varied holders of money and they again spend them... The result of this endless chain is, however, finite as in each of the 'steps' some money will leave the circulation... Thus, in each of the steps, somewhat less money will circulate than in the preceding one... The sum of the series of steps tends to... a certain upper limit. It is just this upper limit that is contained in matrix $R . " 15$

This description evokes the space-time openness condition, which is a solvability condition of our money circulation equation. Though she did not clearly show a mathematical background of her description, it appears to be based on a convergence condition of an infinite series. As mentioned in the preceding section, Augustinovics' equation is denoted $(E-K) b=h$. The inverse of $E-K$ exists if and only if an infinite series $E+K+K^{2}+\cdots$ is convergent, and $(E-K)^{-1}=E+K+K^{2}+\cdots \cdot{ }^{16}$ However, since the author thinks that the infinite series expression is economically worthless, ${ }^{17}$ his opinion is that using the infinite series for the solvability of the money circulation equation is unfavorable from this perspective.

In connection with the solvability, Augustinovics also said:

"The elements of $K$ and the sum of the columns are usually smaller than unity."18

It appears she intended to describe that all column sums of $K$ are less than unity. This is certainly a sufficient condition for the solvability of Augustinovics' money circulation equation. Based on our interpretation of her equation, we explain this fact.

$k_{i j}=\theta_{i} \mu_{i j}$ holds by Equation (26). Therefore, $K$ is a non-negative matrix due to Equations (13) and (16). Further, we can derive

$$
\sum_{j \in N} k_{i j}=\theta_{i} \sum_{j \in N} \mu_{i j} \leq 1 \text { for } \forall i \in N
$$

from Equations (13) and (17). Since $k_{i j}=\theta_{i} \mu_{i j}$ is the $(j$, $i$ )th element of $K$, Equation (27) means that all column sums of $K$ are equal to or less than unity.

Then, if the condition that all column sums of $K$ are less than unity is satisfied, $E-K$ is non-singular as shown by Hermann Minkowski more than one hundred years ago. ${ }^{19}$ This consequent means that Augustinovics' equation has a unique solution.

However, the condition is not necessary for the solvability of her equation. The necessary and sufficient condition is that at least one column sum less than unity exists in any principal submatrix of $K,{ }^{20}$ but Augustinovics did not show it.

On the other hand, this paper has shown a necessary and sufficient condition for the solvability of the money circulation equation. This is a novel contribution of this paper.

Moreover, Augustinovics did not discuss the relationship between the solvability and time irreversibility.

If we denote a subset of $N$ as $F$, the necessary and sufficient solvability condition of Augustinovics' equation can be expressed as $\exists i \in F$ such that $\sum_{j \in F} k_{i j}=\theta_{i} \sum_{j \in F} \mu_{i j}<1$ for $\forall F \subseteq N$. This condition is equivalent to $\exists i \in F$ such that $\theta_{i}<1$ or $\sum_{j \in F} \mu_{i j}<1$ for $\forall F \subseteq N$. If we denote the complement of $F$ as $G$, the latter is equivalent to $\exists i \in F$ such that $\sum_{j \in G} \mu_{i j}>0$ because of Equation (17). Hence, $\exists i \in F$ such that $\Omega_{i}>0$ or $\exists i \in F, \exists j \in G$ such that $Z_{i j}>0$ for $\forall F \subseteq N$ is derived as a necessary and sufficient solvability condition. That is, the space-time openness of all parts is also the solvability condition of her equation. Then, we can see

\footnotetext{
${ }^{15}$ Augustinovics [14] p. 50. In the paper, the matrix $R$ refers to the inverse of $E-K$ (Cf. Augustinovics [14] p.49).

${ }^{16}$ Cf. Nikaido [16] pp. 96-97, Berman \& Plemmons [17] p. 133, Varga [18] pp. 88-89.

${ }^{17}$ Cf. Miura [13] p. 148.

${ }^{18}$ Augustinovics [14] p. 49.

${ }^{19}$ Cf. Berman \& Plemmons [17] p. 161, Plemmons [19] p. 176.

${ }^{20} \mathrm{Cf}$. Theorem 2.17 in Miura [7]. The prototype of this condition was first shown in Beauwens [8].
} 
that this condition is satisfied due to the disposal irreversibility principle by Theorems 3.2 and 3.5. That is, the solvability of her equation is guaranteed by time irreversibility.

This proof requires the recognition of the necessary and sufficient solvability condition. Since Augustinovics did not recognize the condition, it is reasonable that she could not show the proof.

On the other hand, this paper has proved that time irreversibility guarantees the solvability of the money circulation equation. By this proof, the money circulation equation has obtained a firm objective basis. This is also a novel contribution of this paper.

The proof, however, is similar to that of the solvability of the economic input-output equation which the author has stated in the preceding paper. Therefore, some may feel that this paper has not shown any exciting novel findings or inspiring ideas.

Nevertheless, the author thinks that this paper is highly significant because it provides the basis for some intended papers which the author will write in the near future. If you read them, you will notice the significance of this paper.

\section{References}

[1] Newcomb, S. (1966) Principles of Political Economy. A. M. Kelley, New York.

[2] Fisher, I. (1922) The Purchasing Power of Money. New and Revised Edition, the Macmillan Company, New York.

[3] Deane, P. (1968) Petty, William. In: Sills, D.L., Ed., International Encyclopedia of the Social Science, Vol. 12, Crowell Collier and Macmillan, Inc., New York.

[4] Roncaglia, A. (2008) Petty, William. In: Durlauf, S.N. and Blume, L.E., Eds., The New Palgrave Dictionary of Economics, 2nd Edition, Volume 6, Palgrave Macmillan, New York. http://dx.doi.org/10.1057/9780230226203.1278

[5] Theocharis, R.D. (1983) Early Developments in Mathematical Economics. 2nd Edition, The Macmillan Press, London and Basingstoke.

[6] Humphrey, T.M. (1984) Algebraic Quantity Equations before Fisher and Pigou. Economic Review, 13-22. https://www.richmondfed.org/publications/research/economic_review/1984/er700502.cfm

[7] Miura, S. (2014) Non-Singularity Conditions for Two Z-Matrix Types. Advances in Linear Algebra \& Matrix Theory, 4, 109-119. http://dx.doi.org/10.4236/alamt.2014.42009

[8] Beauwens, R. (1976) Semistrict Diagonal Dominance. SIAM Journal on Numerical Analysis, 13, 109-112. http://dx.doi.org/10.1137/0713013

[9] Neumann, M. (1979) A Note on Generalizations of Strict Diagonal Dominance for Real Matrices. Linear Algebra and Its Applications, 26, 3-14. http://dx.doi.org/10.1016/0024-3795(79)90168-X

[10] Varshney, K.R. (2013) Opinion Dynamics with Bounded Confidence in the Bayes Risk Error Divergence Sense. IEEE Conference on Acoustics, Speech and Signal Processing, Vancouver, 26-31 May 2013, 6600-6604.

[11] Shang, Y.L. (2013) Deffuant Model with General Opinion Distributions: First Impression and Critical Confidence Bound. Complexity, 19, 38-49. http://dx.doi.org/10.1002/cplx.21465

[12] Shang, Y.L. (2014) An Agent Based Model for Opinion Dynamics with Random Confidence Threshold. Communications in Nonlinear Science and Numerical Simulation, 19, 3766-3777. http://dx.doi.org/10.1016/j.cnsns.2014.03.033

[13] Miura, S. (2014) Solvability of the Economic Input-Output Equation by Time Irreversibility. Advances in Linear Algebra \& Matrix Theory, 4, 143-155. http://dx.doi.org/10.4236/alamt.2014.43013

[14] Augustinovics, M. (1965) A Model of Money-Circulation. Economics of Planning, 5, 44-57. http://dx.doi.org/10.1007/BF02424905

[15] Leontief, W. and Brody, A. (1993) Money-Flow Computations. Economic Systems Research, 5, 225-233. http://dx.doi.org/10.1080/09535319300000019

[16] Nikaido, H. (1968) Convex Structures and Economic Theory. Academic Press, Cambridge.

[17] Berman, A. and Plemmons, R.J. (1979) Nonnegative Matrices in the Mathematical Sciences. Academic Press, Cambridge.

[18] Varga, R.S. (2000) Matrix Iterative Analysis. 2nd Revised and Expanded Edition, Springer, Berlin. http://dx.doi.org/10.1007/978-3-642-05156-2

[19] Plemmons, R.J. (1977) M-Matrix Characterizations. 1-Nonsingular M-Matrices. Linear Algebra and Its Applications, 18, 175-188. http://dx.doi.org/10.1016/0024-3795(77)90073-8 
Scientific Research Publishing (SCIRP) is one of the largest Open Access journal publishers. It is currently publishing more than 200 open access, online, peer-reviewed journals covering a wide range of academic disciplines. SCIRP serves the worldwide academic communities and contributes to the progress and application of science with its publication.

Other selected journals from SCIRP are listed as below. Submit your manuscript to us via either submit@scirp.org or Online Submission Portal.
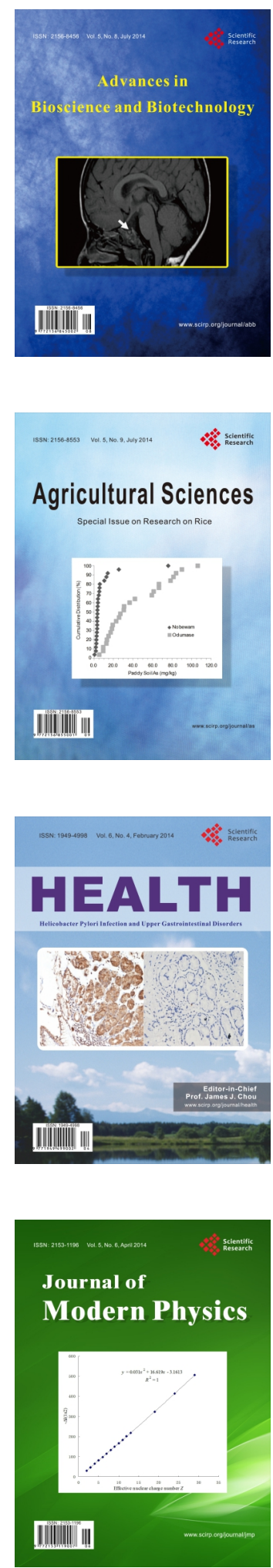
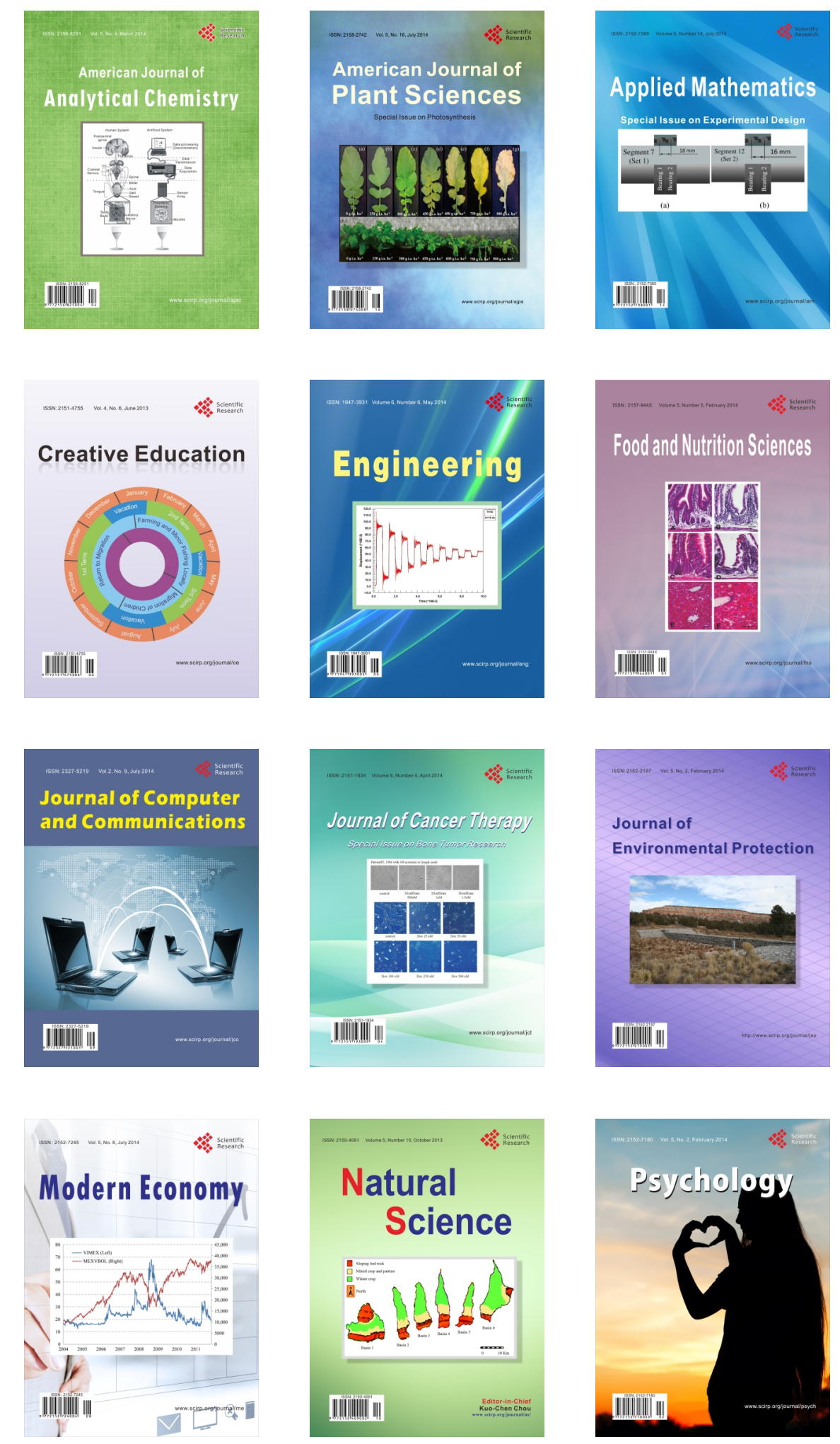